\title{
CD10, CD34 and Ki67 Immunohistochemical Markers Expression in Endometriosis and Adenomyosis
}

\author{
ROXANA-CLEOPATRA PENCIU1*, LILIANA STERIU1, SILVIA IZVORANU ${ }^{1}$, IULIA POSTOLACHE ${ }^{1}$, ANDREI-ADRIAN TICA ${ }^{2}$, \\ DIANA MOCANU ${ }^{1}$, OANA-SORINA TICA ${ }^{3}$, VASILE SARBU ${ }^{4}$, MARIANA DEACU ${ }^{5,6}$, GABRIELA BALTATESCU, ${ }^{6,7}$, IRINA TICA ${ }^{8}$, \\ LUCIAN PETCU9, VLAD-IUSTIN TICA ${ }^{1}$ \\ 'Ovidius University, Department of Obstetrics and Gynecology, Campus, 1 Universitatii Alley, 900470, Constanta, Romania \\ ¿University of Medicine and Pharmacy of Craiova, Faculty of Medicine, Department of Pharmacology, 2 Petru Rares Str., 200349, \\ Craiova, Romania \\ ${ }^{3}$ University of Medicine and Pharmacy of Craiova, Faculty of Medicine, Department of Obstetrics and Gynecology, 2 Petru Rares \\ Str., 200349, Craiova, Romania \\ ${ }^{4}$ Ovidius University Constanta, Department of Surgery,, Campus, 1 Universitatii Alley, 900470, Constanta, Romania \\ ${ }^{5}$ Ovidius University Constanta, Department of Morphopathology, Campus, 1 Universitatii Alley, 900470, Constanta, Romania \\ ${ }^{6}$ Department of Clinical Pathology, St. Apostle Andrew Emergency County Hospital, 145 Tomis Blvd., 900591,Constanta, Romania, \\ 'Ovidius University of Constanta, Faculty of Medicine, Research Center -CEDMOG, 145 Tomis Blvd., 900591, \\ ${ }^{8}$ Ovidius University of Constanta, Department of Internal Medicine, Faculty of Medicine, Campus, 1 Universitatii Alley, 900470, \\ Constanta, Romania \\ ${ }^{9}$ Ovidius University of Constanta, Department of Statistics, Faculty of Dental Medicine, Campus, 1 Universitatii Alley, 900470, \\ Constanta, Romania
}

\begin{abstract}
Endometriosis is a benign disease represented by existence of endometrial tissue outside the uterine cavity. Considered in the past a type of endometriosis, adenomyosis is, presently, described as a possible different entity, comparative to endometriosis. That is the reason why we decided to study two groups of patients one with endometriosis and one with adenomyosis - in order to determine if they are one and the same disease. We included all successive patients admitted and surgically treated in the Emergency Clinical Hospital Constanta between 2015-2017, and, after applying the selection criteria, we assessed 61 patients (group 1) diagnosed with endometriosis - ovarian, cervical, caesarian section scar - and 39 patients (group 2) with adenomyosis. We studied all patients in terms of age, parity, lesions' size, admissions' symptoms, chronic symptoms and immunohistochemical markers CD10, CD34, Ki67. We chose there three markers because of their possible relation to endometriosis and because we were unable to find data regarding the comparison of CD34 or Ki67 expression in endometriosis and adenomyosis and because we did not find articles that reported the expression of these three immunohistochemical markers, combined, for either endometriosis or adenomyosis. According to our study, it seems that endometriosis and adenomyosis are different clinically with regard of age and dysmenorrhea, but there was no statistical difference between the studied immunohistochemical biomarkers' expression in samples of patients with endometriosis or adenomyosis.
\end{abstract}

Keywords: endometriosis, adenomyosis, immunohistochemistry, age, dysmenorrhea

Endometriosis is a benign disease represented by detection of endometrial tissue outside the uterine cavity [1]. It can be found anywhere in the peritoneal cavity: on the ovaries, the fallopian tubes, on the peritoneum, the uterosacral ligaments, the Pouch of Douglas, the rectalvaginal septum and also in caesarian-section scars, laparoscopy or laparotomy scars, on the bladder, bowels, colon, appendix, and rectum [1].

Adenomyosis, considered in the past a type of endometriosis, is represented by the presence of endometrial tissue in the uterine muscle wall. There are three different types of adenomyosis - focal adenomyosis, focal adenomyoma and diffuse adenomyosis. Some of the theories include tissue trauma or some vaginal injury, which determines inflammation and leads to increased macrophages and cytokines which migrate into the uterine myometrium. Another interesting theory would be extension of deep infiltrating endometriosis from outside into the uterine wall [2].

There is, still, a very important question: endometrioma and adenomyoma are the same entity or are they different?
That is the reason why we decided to study two groups of patients - one with endometriosis (all types) and the other one with adenomyosis. We studied the two groups by comparing the immunohistochemistry $(\mathrm{IHC})$ expression of CD10, CD34 and Ki67, together with the patients' age and admission symptoms.

The IHC marker CD10 is known to be expressed by hematopoietic neoplasms like acute lymphoblastic leukemia and follicular lymphomas, normal endometrial stromal cells and endometrial stromal sarcoma [3].

The IHC marker CD34 represents a transmembrane phosphoglycoprotein, which was first identified on hematopoietic stem and progenitor cells and expresses angiogenesis in tissues [3]. [3]

The IHC marker Ki67 is a cellular proliferation marker

We decided to study these three markers considering their relation to endometriosis and to determine if they have the same expression for endometriosis and adenomyosis. Another important reason for this study was that we did notfind articles which have studied all the three biomarkers together for endometriosis and adenomyosis. 


\section{Experimental part}

\section{Material and methods}

In our study we included all successive patients admitted and surgically treated in the Emergency Clinical Hospital Constanta over a period of three years, between 20152017. Sixty-one patients (group 1) were diagnosed with endometriosis - ovarian, cervical, caesarian section scar and 39 patients (group 2) with adenomyosis. We studied all patients in terms of age, parity, lesions' size, admissions' symptoms, chronic symptoms and immunohistochemical markers CD10, CD34, Ki67.

All diagnostics were confirmed by pathology. They were, all, reevaluated by two pathologist and representative samples of each patient were selected for immunohistochemistry. Immunohistochemical tests were performed on four $\mu m$-thick sections of formalin-fixed, paraffin-embedded tissue blocks of cases included in the present study. After the epitope retrieval, tissue sections were incubated with the following antibodies from Biocare Medical (ready-to-use): CD10 (56C6 clone), CD34 (QBEnd 10 clone) and Ki67 (SP6 clone). We used 3,3' diaminobenzidine (DAB) as chromogen, with brown staining. Sections were finally counterstained with Mayer's Haematoxylin. A positive membrane immunostain of stromal cell for CD10 was classified in weak, moderate and strong [4]. CD34 immunostain was considered positive if a distinctive brown color was present in the membrane of the endothelial cells or stromal cells. A positive nuclear reaction for Ki67antibody was considered if a brown staining was noticed in more than 5 cells [5].

Experimental data were analyzed with statistical software IBM SPSS Statistics 23. The procedures used were: descriptive statistics (to characterize discrete and continuous variables defined in the data base), Graphics, Nonparametrical statistical tests (Chi-squared test for association, correlation between two category variables, in order to calculate, in specific circumstances, the risk/ chance ratio $\mathrm{OR}$, and Chi-squared test for the comparison of two proportions) [6-9].

\section{Results and discussions}

The majority of patients from the group with endometriosis were between 30- 40 years (34 patients $55.7 \%$ ), while the respective highest incidence of the patients in the adenomyosis group was in the 40-50 years age interval (30 patients - 76.9\%) (table 1). This was not unusual, as adenomyosis and endometriosis are gynecological diseases that are usually common for

Table 1

PATIENTS' AGE IN THE TWO STUDY GROUPS

\begin{tabular}{|c|c|c|c|c|c|c|c|c|}
\hline & & & & & ige (years) & & & Total \\
\hline & & & {$[20-30)$} & {$[30-40)$} & {$[40-50)$} & {$[50-60)$} & {$[60-70)$} & \\
\hline Group & Endometriosis & Count & 7 & 34 & 18 & 1 & 1 & 61 \\
\hline & & $\%$ within Group & $11.5 \%$ & $55.7 \%$ & $29.5 \%$ & $1.6 \%$ & $1.6 \%$ & $100.0 \%$ \\
\hline & Adenomyosis & Count & 0 & 4 & 30 & 4 & 1 & 39 \\
\hline & & $\%$ within Group & $0.0 \%$ & $10.3 \%$ & $76.9 \%$ & $10.3 \%$ & $2.6 \%$ & $100.0 \%$ \\
\hline Total & & Count & 7 & 38 & 48 & 5 & 2 & 100 \\
\hline & & $\%$ within Group & $7.0 \%$ & $38.0 \%$ & $48.0 \%$ & $5.0 \%$ & $2.0 \%$ & $100.0 \%$ \\
\hline
\end{tabular}

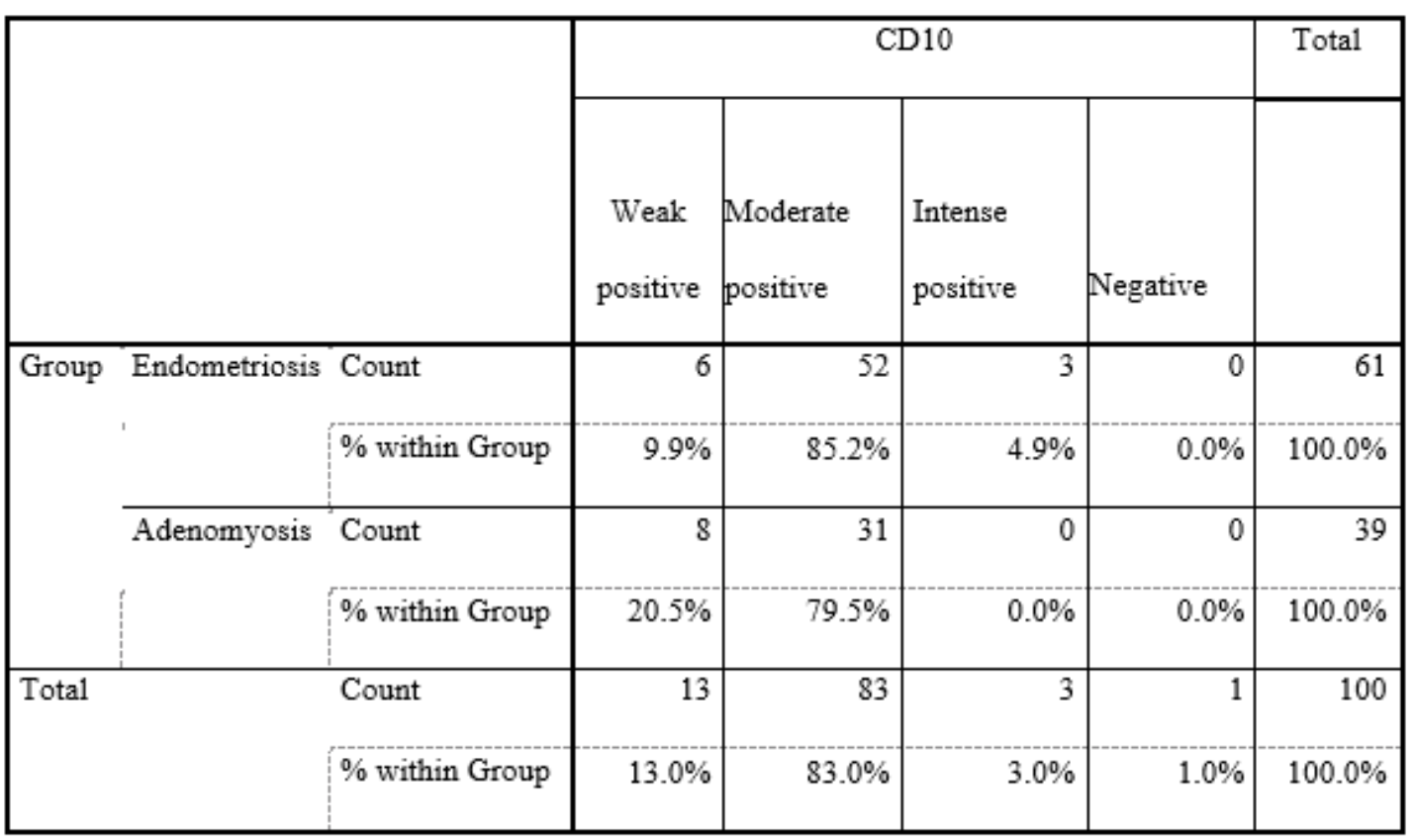

Table 2

CD10

IMMUNOHISTOCHEMICAL BIOMARKER'S EXPRESSION IN SAMPLESFROM PATIENTSWITH ENDOMETRIOSIS OR ADENOMYOSIS 


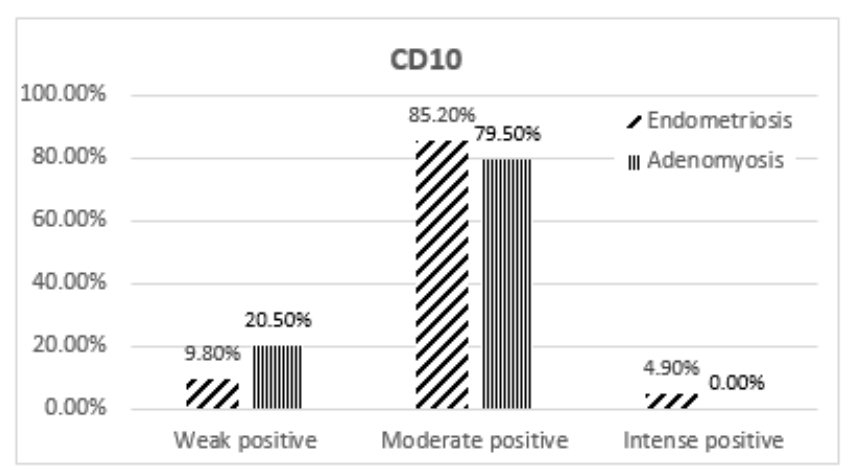

Fig. 1. CD10 immunohistochemical biomarker's expression (\%) in samples from patients with endometriosis or adenomyosis

women of reproductive age. The average age for women with adenomyosis is about 40 years [4].

Patients with endometriosis were younger than patients with adenomyosis. There was a statistical difference concerning the age in the two groups $(p<0.001$, ChiSquare Test). Interestingly, there were 5 patients in menopause in the adenomyosis group and two in the other one.

Thirty patients from group 1 and only 4 from group 2 were infertile. There was a statistical correlation between fertility and each of the two groups. Infertility was more associated to endometriosis than to adenomyosis ( $p=$ $0.001<\alpha=0.05$, Chi-Square Test). Our results are concordant with the available data, as approximately $10 \%$ of women are diagnosed with endometriosis, but usually it appears in infertile women (40\%) [10,11].

The majority of patients from group 2 (35 patients $89.7 \%$ ) had uterine leiomyomas, while only one patient of group 1 was diagnosed with the respective condition.

Dysmenorrhea was mostly associated to group 1, with 42 patients (68.9\%), compared with group 2 , in which only 10 patients $(25.6 \%)$ reported it. There was a statistical correlation between the two parameters - group and dysmenorrhea ( $p<0.001<\alpha=0.05$, Chi-Square Test). There was a calculated possibility of 6.41 times bigger to find a patient with dysmenorrhea in group 1 than in group 2.

In our study, the biomarker CD10 was positive for all the patients, in both groups, thus confirming the presence of endometrial stromal cells $(p=0.143>\alpha=0.05$, ChiSquare Test) (table 2, fig. 1). It was moderately positive in
52 patients (85.2\%) of group 1 and 31 patients (79.5\%) of group 2. There was no statistical correlation between the degree of positivity of CD10 and the size of endometriotic or adenomyotic lesions.

Our data were logically relevant and confirm the correctness of the inclusion process, as the immunohistochemical marker CD10 is largely expressed by stromal endometrial cells located outside the uterus. It can certify the diagnosis of endometriosis [4]. It can be also used for women with minimal disease, to confirm the diagnosis [12].

IHC biomarker CD34 was moderately positive in the stromal cells of 18 patients (29.5\%) in group 1 and in 7 patients (17.9\%) in group 2. It was negative in 43 patients $(70.5 \%)$ in group 1 and 31 patients (79.5\%) in group 2 (table 3 , fig. 2). There was no statistical difference between the two groups ( $p=0.213>\alpha=0.05$, Chi-Square Test).

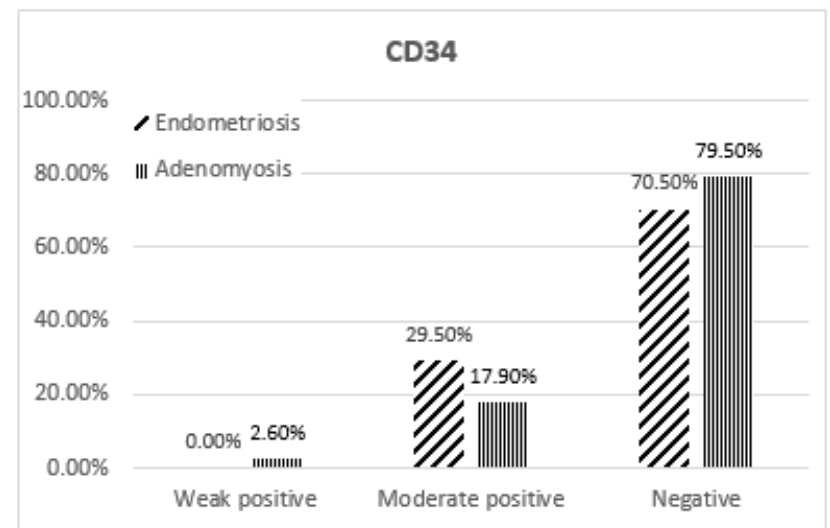

Fig. 2. CD34 immunohistochemical biomarker's expression in samples from patients with endometriosis or adenomyosis

Immunohistochemical marker CD34 is used to identify endothelial cells. Women with endometriosis have an increased cell proliferation in their endometrium. This suggests that the respective endometrium can implant itself and survive in ectopic locations, outside the uterine cavity [13]. Meenakshi M et al. observed a phenomenon of vascular involvement in adenomyosis. When it is widespread, a neoplastic process may be considered. This vascular pattern may sustain a new theory of developing adenomyosis from the cells that are intimately situated to myometrial blood vessels, probably multipotential perivascular cells [14]. The important relation of CD34 with

Table 3

CD34 IMMUNOHISTOCHEMICAL BIOMARKER'S EXPRESSION IN PATIENTS WITH ENDOMETRIOSIS OR ADENOMYOSIS

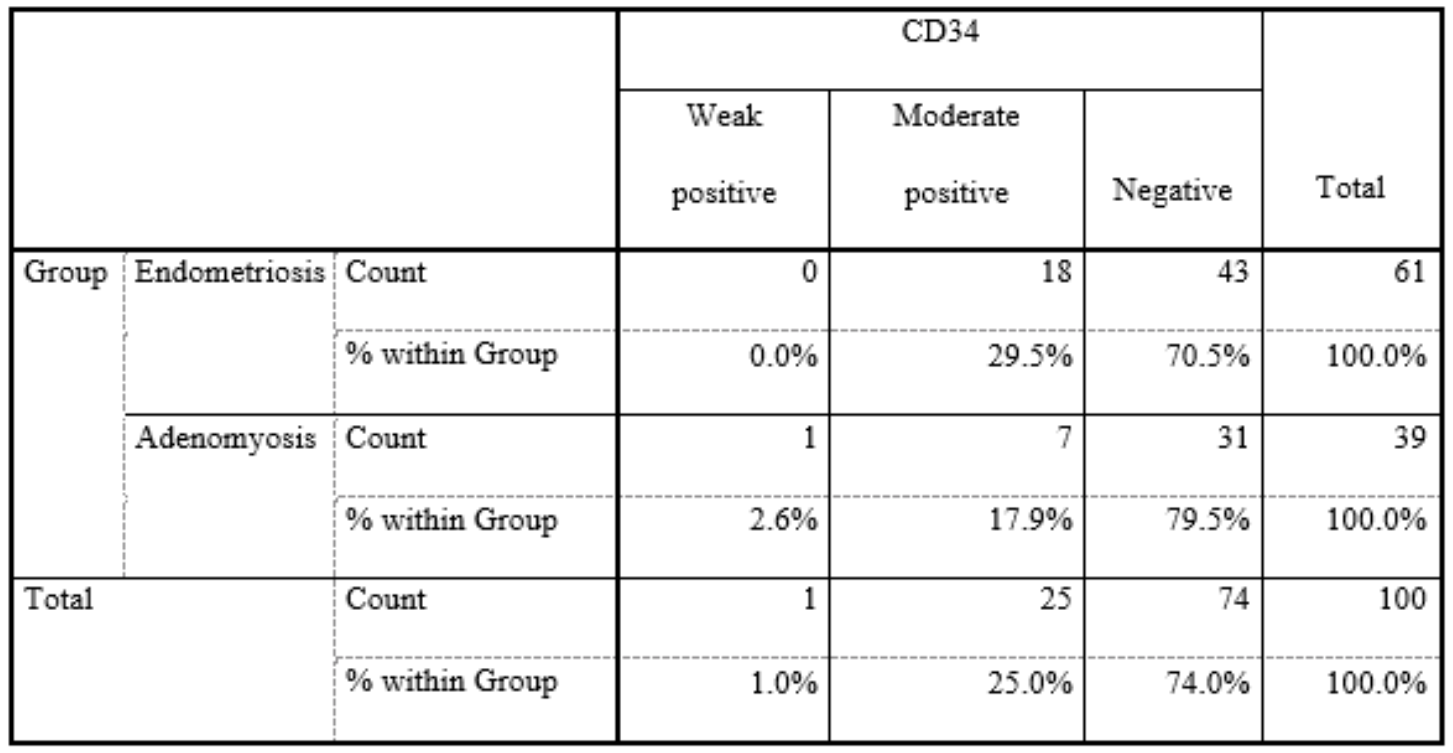




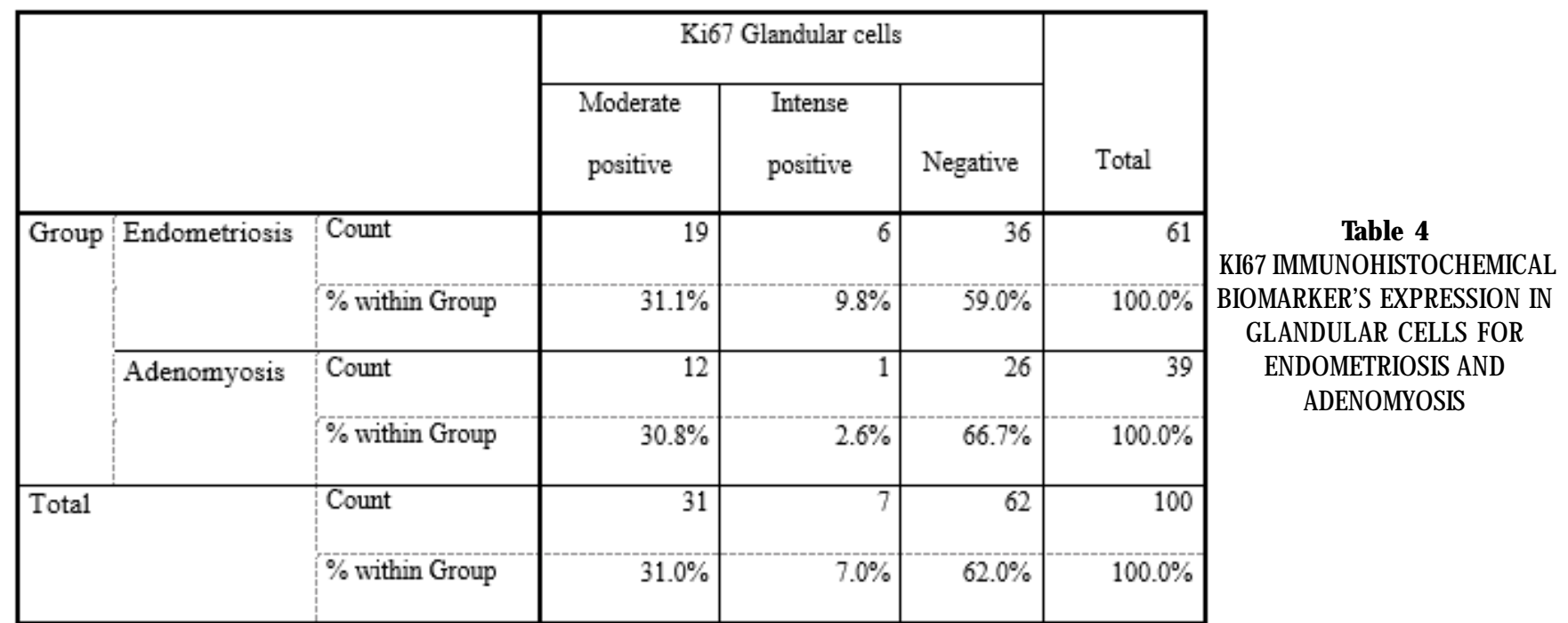

endometriosis and adenomyosis was the reason why we decided to determine if there is a correlation between the two groups. We could not find in the literature a study comparing the expression of this immunohistochemical marker in-between endometriosis and adenomyosis.

For group 1, IHC biomarker Ki67 (in glandular cells) was positive in 25 patients (40.9\%) and negative in 36 patients (59.0\%). Patients in group 2 expressed almost the same proportion - 13 patients positive (33.4\%) and 26 patients negative (66.7\%) (table 4, fig. 3). There was no significative difference, on this regard, between the two groups ( $p=$ $0.213>\alpha=0.05$, Chi-Square Tests) (fig. 3).

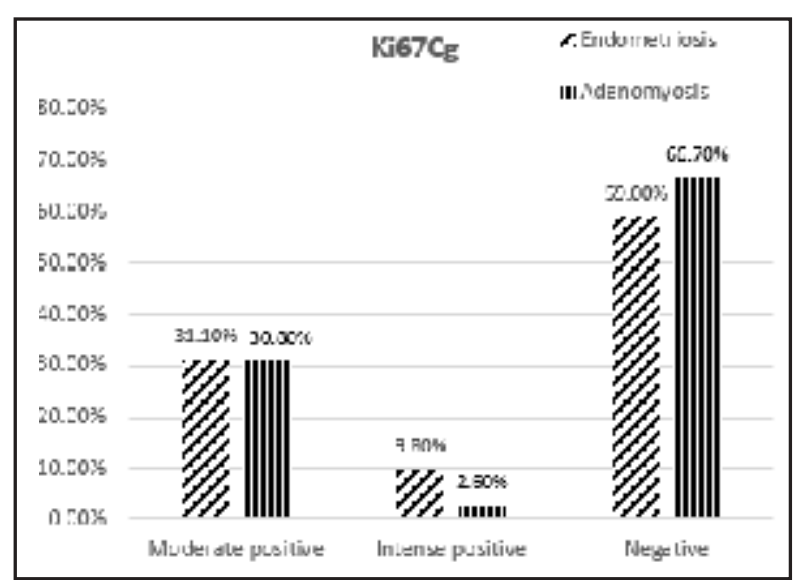

Fig. 3. Ki67 glandular (Ki67 Cg) immunohistochemical biomarker's expression in samples from patients with endometriosis or adenomyosis
The biomarker Ki67 in stromal cells was moderate positive in 49 patients (80.3\%) and negative in 7 patients $(11.5 \%)$ in group 1. In group 2 patients, it was moderate positive for 37 patients (94.9\%) and negative in one patient (2.6\%) (table 5, fig. 4). There was no statistical difference between the two groups for IHC Ki67 in stromal cells ( $p=$ $0.052>\alpha=0.05$, Chi-Square Tests) (fig. 4).

Jehn-Hsiahn Yang et al. observed that a high Ki67 index in immunohistochemistry can be predictive for developing adenomyosis [15]. On the other hand, Matsumoto $Y$ et al. studied the bcl-2 gene expression for apoptosis and Ki67 expression as a proliferative marker. They observed that

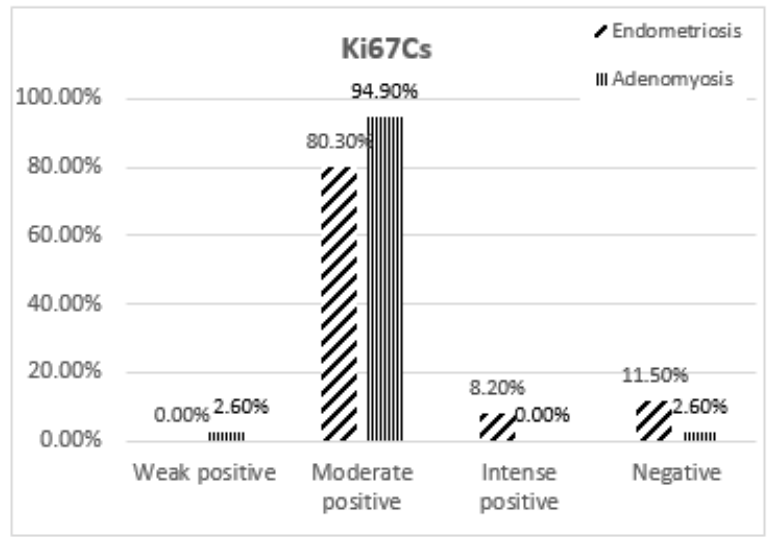

Fig. 4.Ki67 stromal (Ki67 Cs) immunohistochemical biomarker's expression in samples from patients with endometriosis or adenomyosis

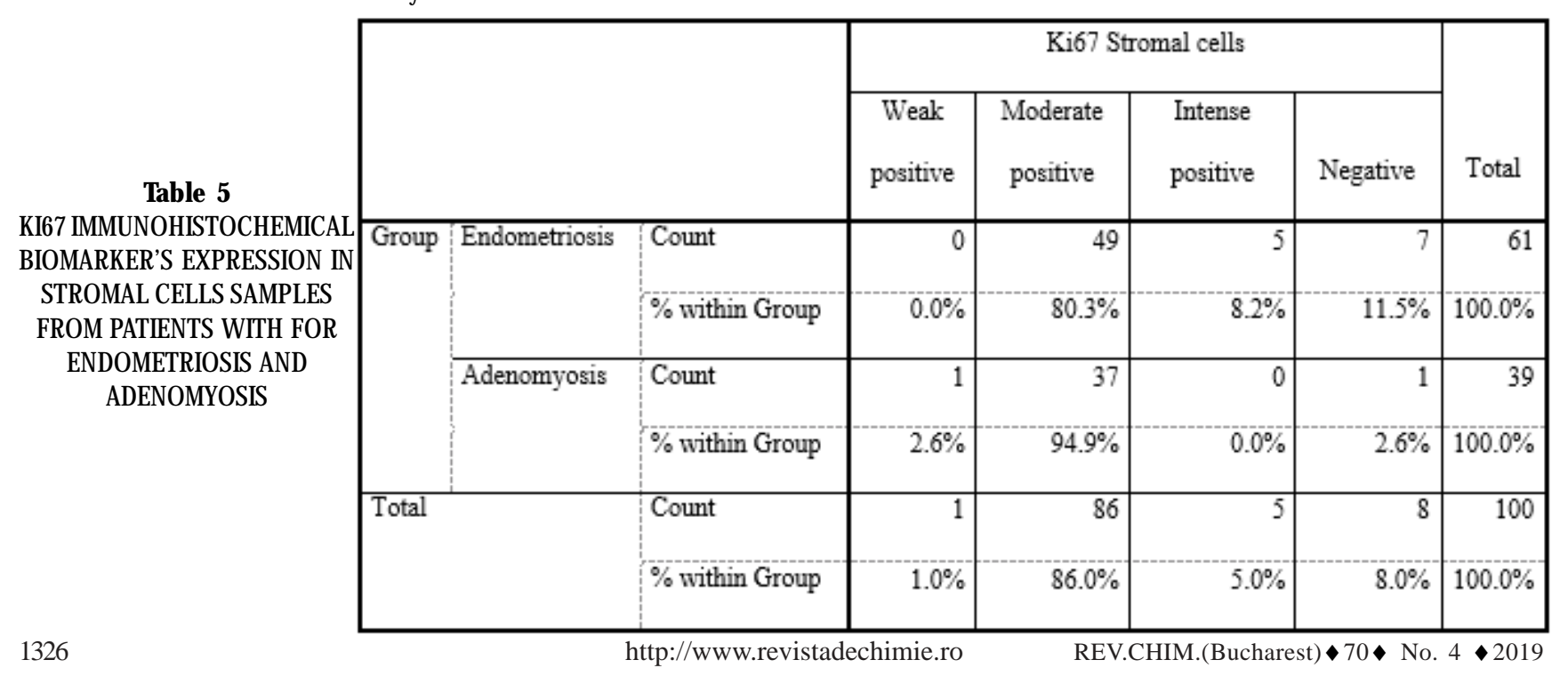


Ki67 was expressed by glandular epithelium of ectopic endometrium, without correlation to menstrual phases. In the secretory phase, this immunohistochemical marker was less expressed by eutopic endometrium in functional and basal layers. This is the reason why they concluded that adenomyotic lesions have no origin in the basal endometrium [16]. Because of these data we decided to study the IHC biomarker in both glandular and stromal cells.

As for CD34, we could not find in the literature a study comparing the expression of Ki67 immunohistochemical marker in-between endometriosis and adenomyosis.

Neither could we find data concerning the concomitant expression of CD10, CD34 and Ki67 either for endometriosis nor for adenomyosis. This leads to the logical assertion of the non-available comparison of endometriosis and adenomyosis regarding the bundle CD10, CD34 and Ki67.

\section{Conclusions}

Although, in our study, there were significant clinical statistical differences in-between groups with regard to age and dysmenorrhea, immunohistochemical markers CD10, CD34 and Ki67 (glandular or stromal) were similarly expressed in the samples from patients with endometriosis or adenomyosis.

We did not find articles that studied the expression of these three immunohistochemical markers, combined, for either endometriosis or adenomyosis. We were, equally, unable to find data regarding the comparison of CD34 or Ki67 expression in endometriosis or adenomyosis.

Acknowledgments: This research was performed in the Center for Research and Development of the Morphological and Genetic Studies of Malignant Pathology from the Ovidius University of Constanpa, POSCCE 2.2.1. Project ID: 1844, code SMIS: 48750, CEDMOG, contract 627/11.03.2014.

\section{References}

1. BROSENS, I., BENAGIANO, G. History of endometriosis. In: GIUDICE, L. C., EVERS J LH, HEALY DL. Endometriosis Science and Practice: Ed Blackwell Publishing Ltd, 2012; p 3-18.

2.LEYENDECKER, G., WILDT, L. Uterine Peristalsis and the Development of Endometriosis and Adenomyosis. In: GIUDICE, L. C., EVERS J LH, HEALY DL. Endometriosis Science and Practice, Ed Blackwell Publishing Ltd, 2012, p 200-211.
3.STUART,L.N.CD34.PathologyOutlines.comw ebsite.http:// www. pathologyoutlines.com/topic/cdmarkerscd34.html.

4.SUMATHI, V.P, MCCLUGGAGE, W.G. CD10 is useful in demonstrating endometrial stroma at ectopic sites and in confirming a diagnosis of endometriosis. J Clin Pathol. 2002 May; 55(5): 391-392.

5.KAHYAOGLU, I., KAHYAOGLU, S., MORALOGLU, O., ZERGEROGLU, S., SUT, N., BATIOGLU, S. Comparison of Ki-67 proliferative index between eutopic and ectopic endometrium: a case control study. Taiwan J Obstet Gynecol, 2012, 51(3):393-396.

6.BEGU, L. Statistica si software statistic, Ed. Clauet, Bucuresti, 1999 7.PETCU, L.C. Analiza statistica cu SPSS-Note de Curs, Ed. Ovidius University Press, Constanta, 2011, p.1-303

8.LUPU, G., PETCU, L.C., LUPU, E.C. Matematici aplicate si Biostatistica, Ed. Virom, Constanta, 2006, p.221-293

9.POPA, M. Statistici multivariate - Aplicatii în psihologie, Ed. Polirom, Iasi 2010

10.RAPKIN, A.J., NATHAN, L. Pelvic pain and dysmenorrhea. In: BEREK, J.S., NOVAK E., editors. Berek \& Novak's gynecology. 15th ed. Philadelphia (PA): Lippincott Williams \& Wilkins; 2012. pp. 470-504. 11. HEMMINGS, R., RIVARD, M., OLIVE, D.L., POLIQUIN-FLEURY, J., GAGNE, D., HUGO, P., et al. Evaluation of risk factors associated with endometriosis. Fertil Steril. 2004;81:1513-1521

12. POTLOG-NAHARI, C., FELDMAN, A.L., STRATTON, P., KOZIOL, D.E., SEGARS, J., MERINO, M.J., NIEMAN, L.K. CD10 immunohistochemical staining enhances the histological detection of endometriosis. Fertil Steril. 2004 J ul; 82(1):86-92.

13.W INGFIELD, M., MACPHERSON, A., HEALY, D.L., ROGERS, P.A. Cell proliferation is increased in the endometrium of women with endometriosis. Fertil Steril. 1995 Aug; 64(2):340-346.

14.MEENAKSHI, M., MCCLUGGAGE, W.G. Vascular involvement in adenomyosis: report of a large series of a common phenomenon with observations on the pathogenesis of adenomyosis. Int J Gynecol Pathol. 2010 Mar; 29(2):117-21.

15.YANG, J.H, WU, M.Y., CHEN, C.D., CHEN, M.J., YANG, Y.S., HO, H.N. Altered apoptosis and proliferation in endometrial stromal cells of women with adenomyosis. Human Reproduction, Volume 22, Issue 4, 1 April 2007: 945-952.

16.MATSUMOTO, Y., IWASAKA, T., YAMASAKI, F., SUGIMORI, H. Apoptosis and $\mathrm{Ki}-67$ expression in adenomyotic lesions and in the corresponding eutopic endometrium. Obstet Gynecol. 1999; 94(1):71.

$\overline{\text { Manuscript received: } 28.11 .2018}$ 